Journal of Case Reports 2019;9(2):132-134

\title{
West syndrome associated with Multiple Neurodevelopmental Co-morbidities
}

\author{
Yogender Kumar Malik, Akhilesh Sharma, Susanta Kumar Padhy, Poonam Adlakha \\ Department of Psychiatry; Postgraduate Institute of Medical Education and Research, Chandigarh, India.
}

\section{Corresponding Author:}

Dr. Yogender Kumar Malik

Email: yogendermalik187@gmail.com

This is an Open Access article distributed under the terms of the Creative Commons Attribution License (creativecommons.org/ licenses/by/3.0).

Received : May 1, 2019

Accepted : June 19,2019

Published : June 25, 2019

\begin{abstract}
Background: West syndrome (WS) is one of the most common causes of refractory epilepsy with infantile onset and characterized by epileptic spasms and neurodevelopmental problems. About two third of children with West syndrome have mental retardation while significant number have autism spectrum disorders and hyperkinetic disorder. Case Report: The index case describes a child with West syndrome where all three neurodevelopment disorders are present along with epileptic spasms and associated abnormal electroencephalogram. Conclusion: It is important to examine a child presenting with such a plethora of symptoms in a holistic way, as early as possible, so that we can prevent the associated co-morbidities related to neurodevelopment process and untreated epileptic spasms.
\end{abstract}

Keywords: Autism Spectrum Disorder, Electroencephalography, Infantile Spasms, Neurodevelopmental Disorders.

\section{Introduction}

West syndrome (WS) is one of the most common causes of refractory epilepsy with infantile onset and characterized by epileptic spasms, neurodevelopmental problems due to cessation and regression of development and common association with electroencephalogram finding of hypsarrhythmia [1,2]. The association of neurodevelopment disorders like intellectual disability, autism spectrum disorder and attention deficit hyperkinetic disorder with infantile spasms is well known. This association occurs in varying combination and severity of epilepsy and various neurodevelopment disorders [3]. About 70-80\% of children with West syndrome have mental retardation, $10-13 \%$ have autism spectrum disorders and $12-15 \%$ has hyperkinetic disorder [4]. Studies also reported that in children diagnosed with epilepsy in the first year of life, autism spectrum disorder developed in 14\% while it developed in $46 \%$ of children diagnosed with West syndrome $[5,6]$. The review of literature showed only $16 \%$ of infantile spasms cases had normal development [6]. We hereby report a case of West syndrome associated with multiple neurodevelopmental co-morbidities.

\section{Case Report}

This case concerns a male child (age 4 years and 6 months) born out of preterm vaginal delivery at 8 completed months with no other perinatal complications but was large for gestational age (3.5 kg). He was achieving developmental milestones normally till first 5 months of age. He developed epileptic spasms at this age and was diagnosed to have West syndrome with characteristic EEG finding of hypsarrhythmia. MRI brain, metabolic work up and spectroscopy were unremarkable. He was treated with intramuscular adrenocorticotropic hormone therapy for 10 weeks and oral valproate from the age of 9-10 months to 4 years [4]. After 1st year of life till 4 years of age he was free of spasms. For next 3 years oral sodium valproate was continued. From 6 month 
to one year of age, he lost the social and speech milestones but gross motor, fine motor progressed slowly. From one year onwards he did progressed in all the milestones but there was atypicality in socialization and communication both verbal as well as non verbal along with speech delay. He also had restricted repetitive verbal and motor behaviors, hyperactivity, impulsivity and selfinjurious behavior. His practical, cognitive and adaptive skills were like of a 3 year old child when he was 4 and half years of age $(\mathrm{IQ}=67)$. All the behavioral issues were more than expected for his mental age. But he was seizure free and going to school with difficulties present both at home and school. His antiepileptic medications were stopped at 4 years; thereafter his behavioral problems started worsening. He was treated with multiple antipsychotics by pediatrician but with no response and referred to Psychiatry outpatient department. When assessed by psychiatrist, mother's report of episodes of vacant staring, repeat EEG was performed, which revealed inter-ictal sharp waves. Valproate was restarted with significant improvement. Along with speech therapy, occupational therapy and parent management training for challenging behaviors were started.

\section{Discussion}

West syndrome primarily occurs in infantile age and usually recognized at 3-15 months of life. In addition to clinical features of flexors or extensors spasms, hypsarrhythmias on EEG is most commonly associated finding. The treatment options include adrenocortico hormone therapy, oral steroids or vigabatrin [7]. Those cases which have associated risk factors in prenatal, postnatal and perinatal period are called as symptomatic cases and those cases where no such factor or developmental delay is present before the onset of infantile spasm are called idiopathic. The symptomatic cases have poorer prognosis in comparison to idiopathic cases. Similarly neurodevelopmental disorders like autism spectrum disorders, ADHD and intellectual disability is

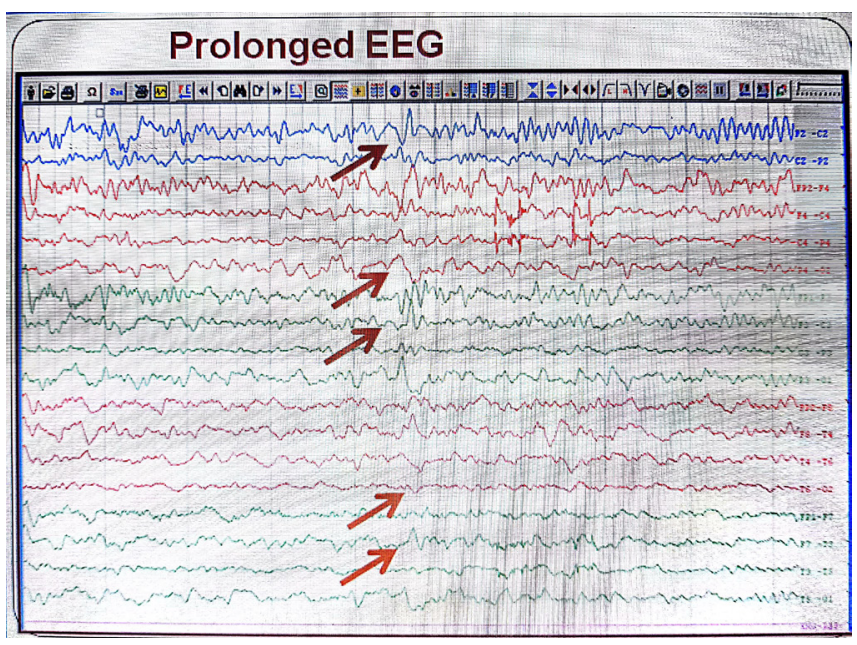

Fig.1: EEG showed sharp inter-ictal waves.

frequently associated with the prenatal, perinatal and postnatal risk factors. There is significant gap in knowledge, attitude and practices among caregivers on this disorder as observed in a recent survey [8]. There is increased incidence of epilepsy in general with all the three neuro developmental disorders. The epileptic spasms specifically have a possible association with autism $[9,10]$.

In index case risk factors like premature rupture of membrane leading to preterm birth, large for date neonate were present but no developmental delays or developmental atypicalities were present in first five months of infancy during which development was normal. In index case infantile spasms appeared typically before the nine months i.e around $5^{\text {th }}$ month. Developmental regression in social and communication milestones along with delay in all other milestones started after the appearance of infantile spasms. After control of infantile spasms the problems in social and communication milestones started appearing as characteristics of autism spectrum like reversal of pronouns, lack of eye contact, motor stereotypes, restrictive repetitive behaviors, delayed and stereotyped speech with minimal development of language as per mental age. After stopping the valproate along with these problems, symptoms of hyperactivity, inattention and impulsivity also 
worsened to an extent which could not be accounted for by intellectual disability and autism spectrum disorder which he was already manifesting with leading to an independent psychiatric diagnosis of ADHD in addition to mild intellectual disability and autism spectrum disorder.

\section{Conclusion}

The present case has a distinction where all three neurodevelopmental disorders i.e. intellectual disability, autism spectrum and ADHD were found as co-morbidities in a case of epileptic spasms with characteristic electroencephalographic (EEG) finding presenting as phenotype of West syndrome. It is important to recognize the epileptic syndrome as early as possible so that we can prevent the associated co-morbidities related to neurodevelopment process and untreated epileptic spasms.

Contributors: YKM participated in the patient care, and manuscript writing; AS and SKP participated in study conception and writing of the paper; PA participated in revising the manuscript critically. YKM will act as guarantor. All authors approved the final version of this manuscript. Funding: None; Competing interests: None stated.

\section{References}

1. Wilmshurst JM, Ibekwe RC, O Callaghan FJK. Epileptic spasms. Trying to teach old dog new tricks. Seizure. 2017;44:81-86.
2. Hrachovy RA, Frost JD. Infantile epileptic encephalopathy with hypsarrytmia. J Clin Neurophysiol. 2003;20:408-425.

3. Nickels KC, Zaccariello MJ, Hamiwka LD, Wirrell EC. Cognitive and neurodevelopmental comorbidities in paediatric epilepsy. Nat Rev Neurol. 2016;12:465-476.

4. Seamundsen E, Ludvingsson P, Rafnsson V. Autism spectrum disorders in children with a history of infantile spasms: a population based study. J Child Neurol. 2007;22:1102-1107.

5. Riikonen R. A long-term follow-up study of 214 children with the syndrome of infantile spasms. Neuropediatrics. 1982;13:14-23.

6. Singhi P, Ray M. Profile of West syndrome in North Indian children. Brain Dev. 2005;27:135-140.

7. Sahu JK. Infantile spasms-evidence based medical management. Indian J Pediatr. 2014;81:1052-1055.

8. Vaddi VK, Sahu JK, Dhawan SR, Suthar R, Sankhyan N. Knowledge, attitude and practice (KAP) study of pediatricians on infantile spasms. Indian J Pediatr. 2018; 85:836-840.

9. Williams AE, Giust JM, Kronenberger WG, Dunn DW. Epilepsy and attention-deficit hyperactivity disorder: links, risks, and challenges. Neuropsychiatr Dis Treat. 2016;12:287-296.

10. Berg AT, Plioplys S, Tuchman R. Risk and correlates of autism spectrum disorder in children with epilepsy: a community-based study. J Child Neurol. 2011;26:540547. 